\title{
The temporal stability and maintenance mechanisms of alpine meadow communities under clipping and fertilization
}

\author{
Ting Wang ${ }^{1}$, Chenglong Guo ${ }^{1}$, Silin Sang ${ }^{1}$, Yiting Liu ${ }^{1}$, Gang Liu ${ }^{2}$, Desheng $\mathrm{Qi}^{3}$, and \\ Zhihong $\mathrm{Zhu}^{2}$ \\ ${ }^{1}$ Shaanxi Normal University College of Life Sciences \\ ${ }^{2}$ Shaanxi Normal University \\ ${ }^{3}$ Qinghai Normal University
}

February 3, 2021

\begin{abstract}
The effects of human disturbance on the stability of alpine meadow communities, their diversity-stability relationship, and the underlying mechanisms are still not fully understood. Here, we performed a 12-year-long (2007-2018) two-factor $(2 \times 3)$ controlled experiment on Kobresia humilis on the Tibetan Plateau. The manipulations included three clipping levels (no clipping, NC; moderate clipping, MC; heavy clipping, HC) and two fertilization levels (no fertilization, NF; fertilization, F). Our results revealed that the two clipping manipulations significantly increased the temporal stability of alpine meadow communities, whose significant increase was more pronounced under the MC than HC treatment. Moreover, asynchrony effects, portfolio effects, and facilitation interactions were all present in the communities under the six types of experimental treatment combinations. Additionally, a selection effect was detected in the compound communities, demonstrating characteristics that are common to different mechanisms. There were no significant differences in the effects of these mechanisms on community temporal stability between the $\mathrm{NC}-\mathrm{NF}$ and $\mathrm{MC}-\mathrm{NF}$ interactive communities. The portfolio effects predominated when clipping intensity was moderate under both fertilization and non-fertilization conditions. By contrast, in the compound communities, the selection effect predominated. In summary, we conclude that in meadow communities that undergo clipping and fertilization disturbances, facilitation interactions and weak interactions make a greater contribution toward maintaining their temporal stability.
\end{abstract}

\section{Hosted file}

Main Document_WangTing.pdf available at https://authorea.com/users/393491/articles/507094the-temporal-stability-and-maintenance-mechanisms-of-alpine-meadow-communities-underclipping-and-fertilization 


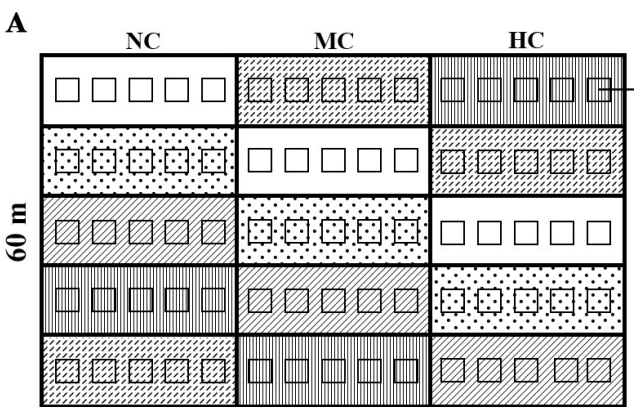

$100 \mathrm{~m}$

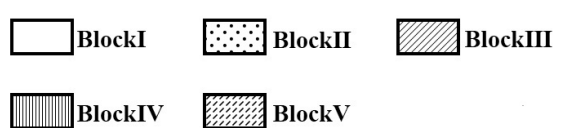

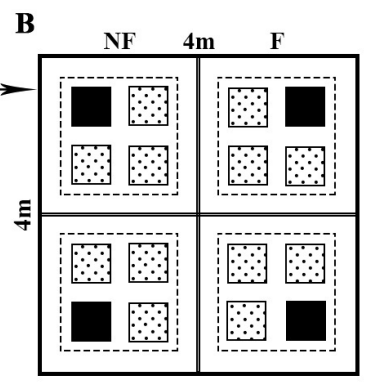

Permament quadrat $(0.5 \mathrm{~m} \times 0.5 \mathrm{~m})$

$\because \because$ Sampling quadrat

$\because(0.5 \mathrm{~m} \times 0.5 \mathrm{~m})$

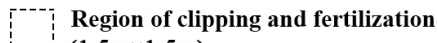
$(1.5 \mathrm{~m} \times 1.5 \mathrm{~m})$
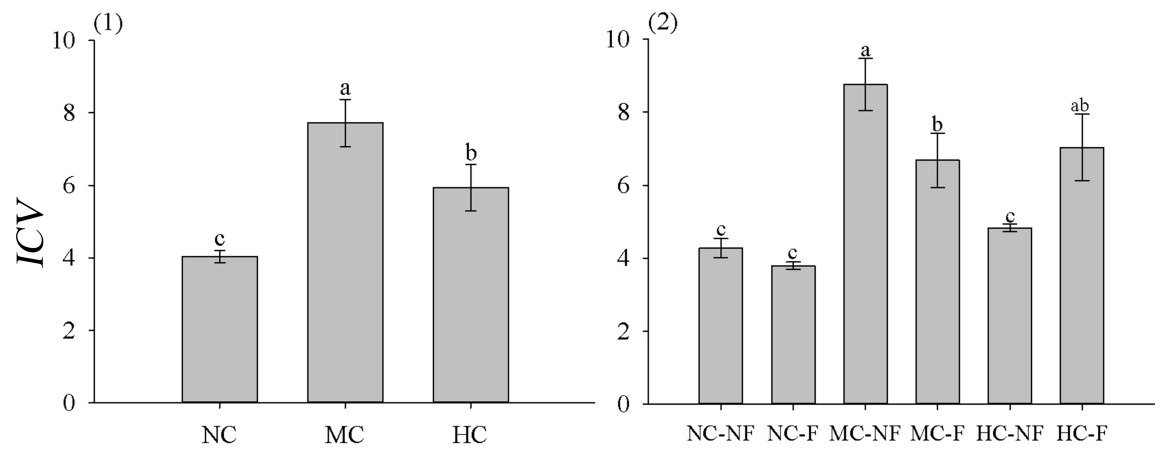

Experimental treatment

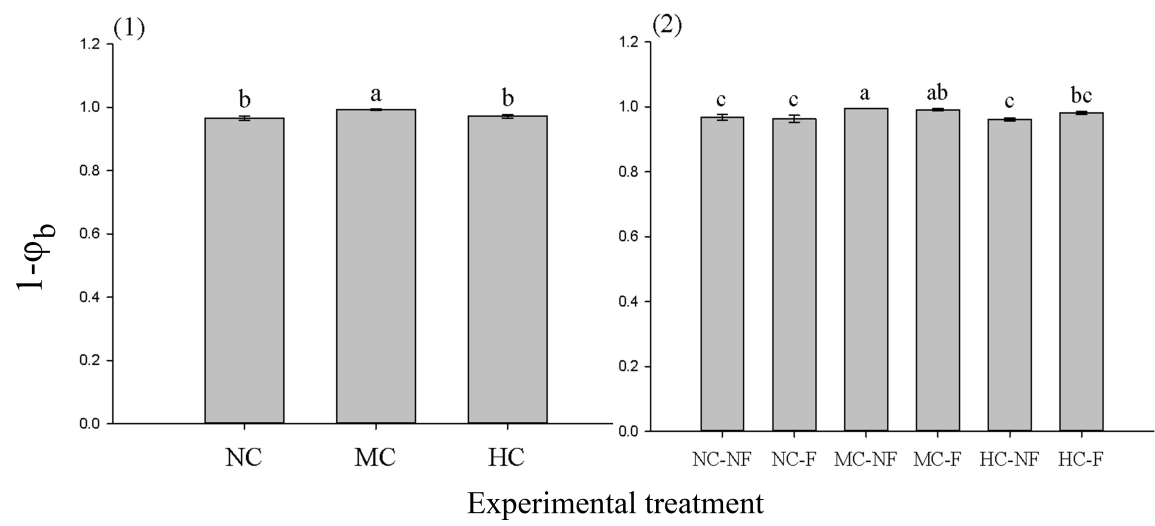



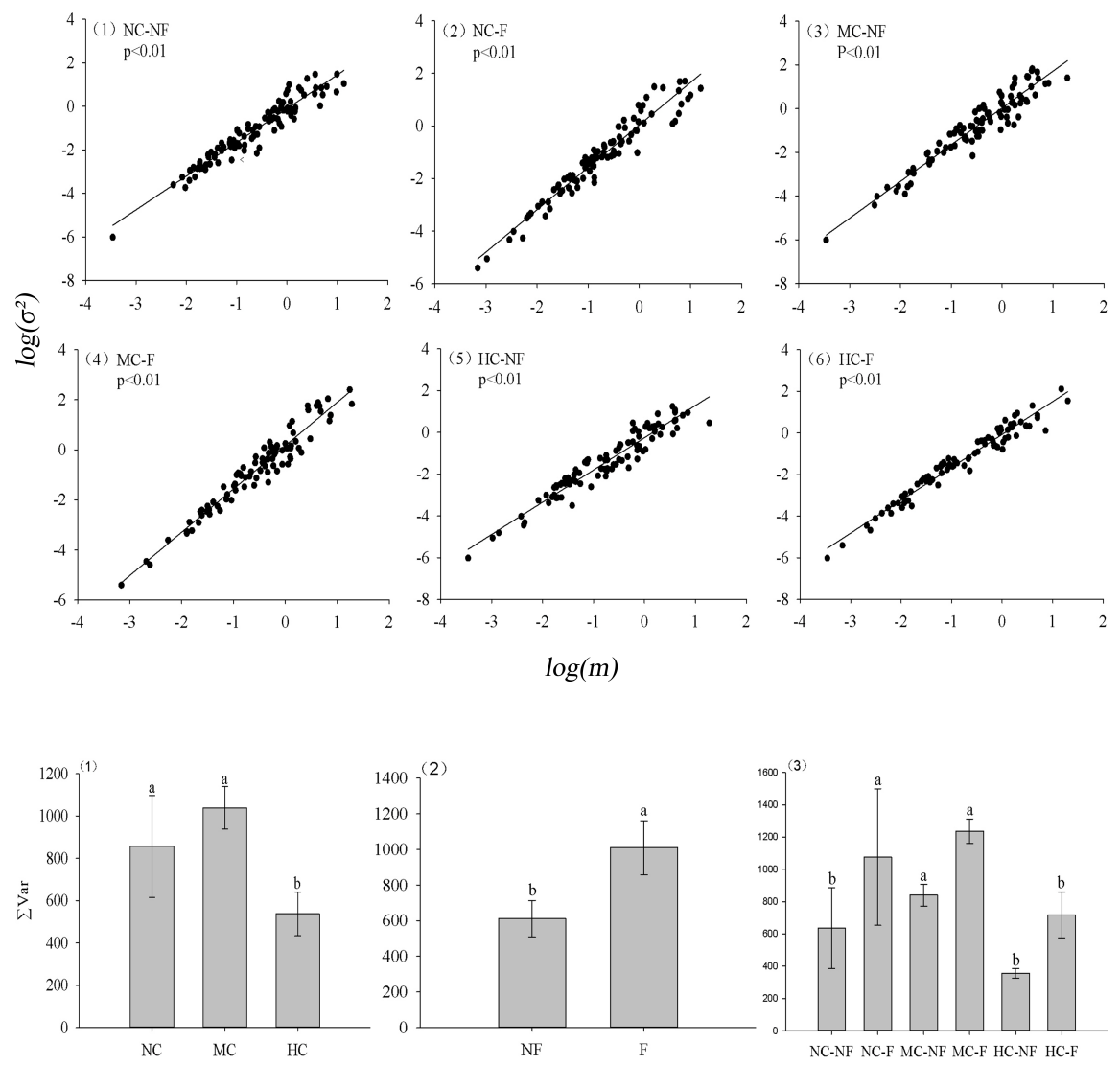

Experimental treatment
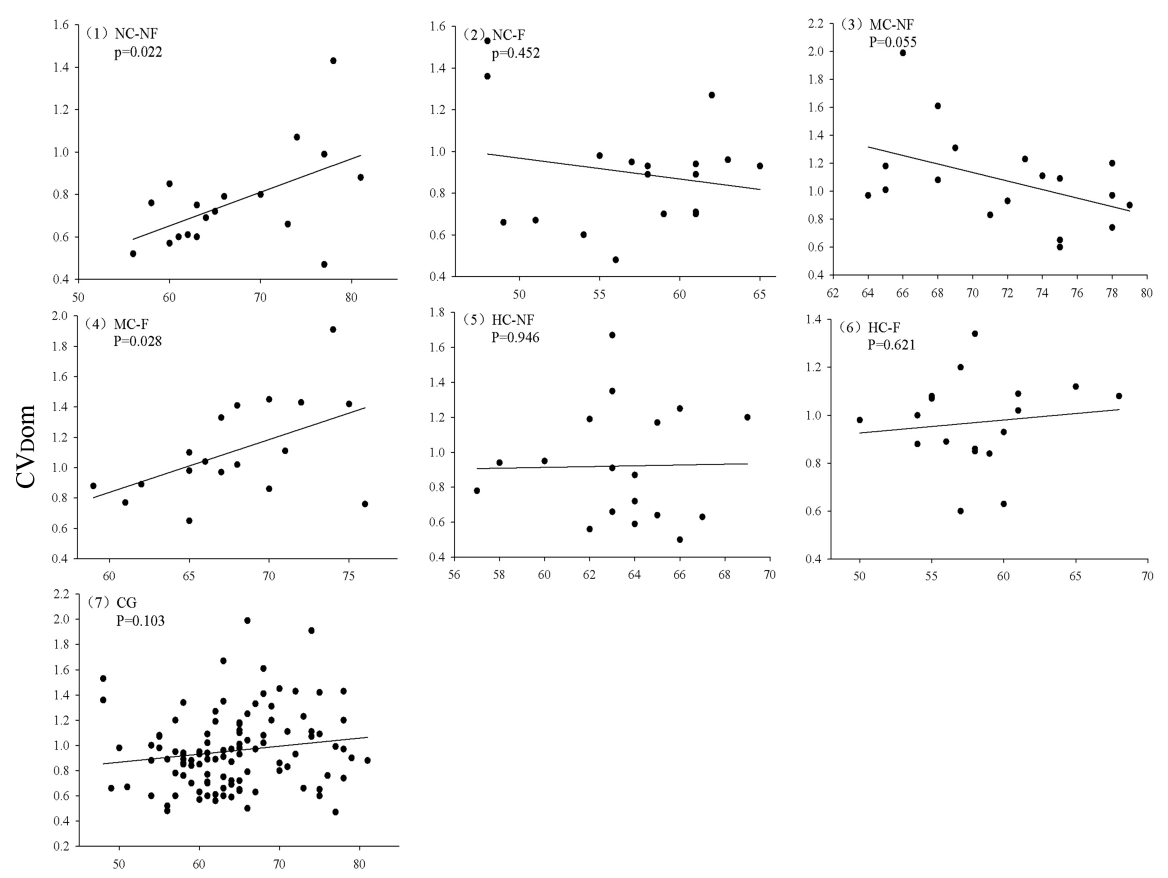

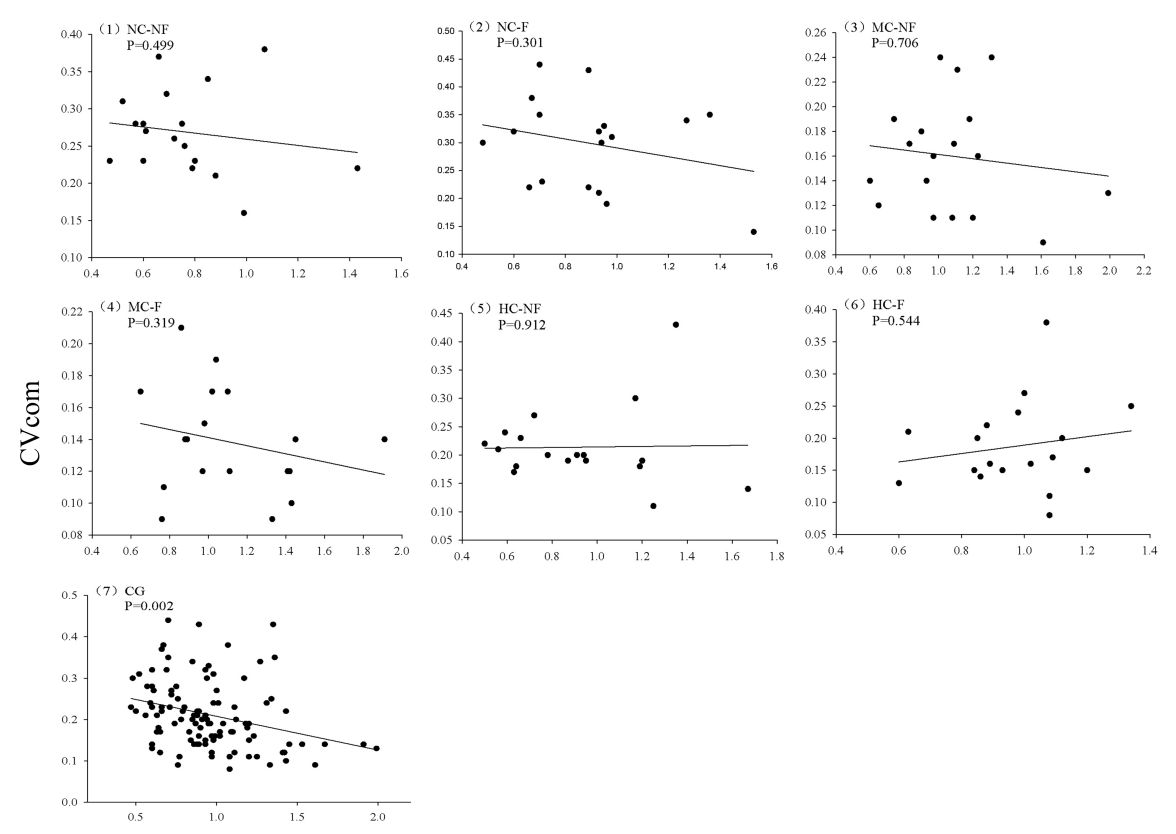

CVDom
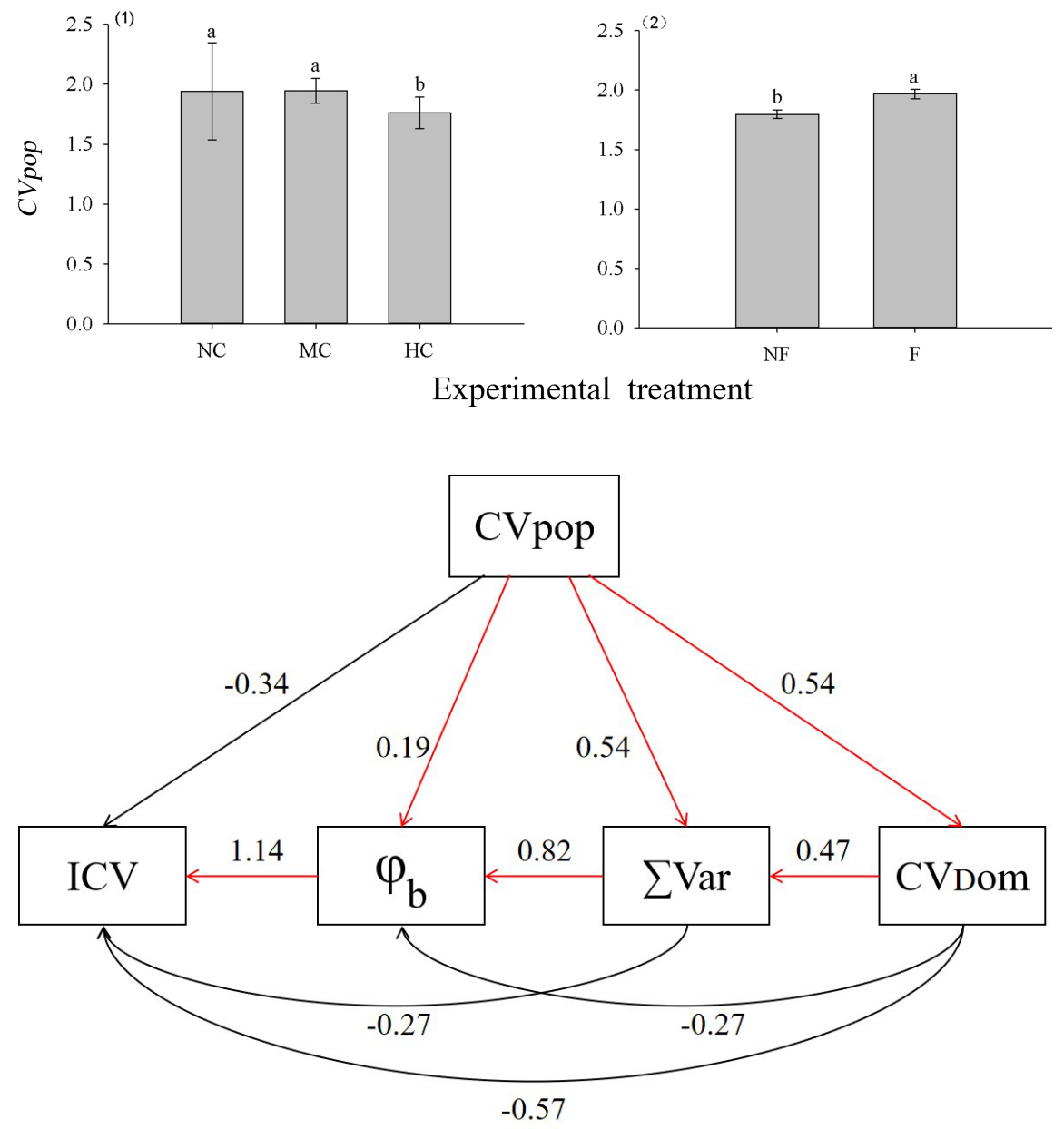